\title{
The redshift evolution of bias and baryonic matter distribution
}

\author{
P. Valageas ${ }^{1}$, J. Silk ${ }^{2,3}$, and R. Schaeffer ${ }^{1}$ \\ 1 Service de Physique Théorique, CEA Saclay, 91191 Gif-sur-Yvette, France \\ 2 Astrophysics, Department of Physics, Keble Road, Oxford OX1 3RH, UK \\ 3 Institut d'Astrophysique de Paris, CNRS, 98bis Boulevard Arago, 75014 Paris, France
}

Received 12 January 2000 / Accepted 13 November 2000

\begin{abstract}
We study the distribution of baryonic and luminous matter within the framework of a hierarchical scenario. Using an analytical model for structure formation which has already been checked against observations for galaxies, Lyman- $\alpha$ clouds, clusters and reionization processes, we present its predictions for the bias of these objects. We describe its dependence on the luminosity (for galaxies or quasars) or the column density (for Lyman- $\alpha$ absorbers) of the considered objects. We also study its redshift evolution, which can exhibit an intricate behaviour. These astrophysical objects do not trace the dark matter density field, the Lyman- $\alpha$ forest clouds being undercorrelated and the bright galaxies overcorrelated, while the intermediate class of Lyman-limit systems is seen to sample the matter field quite well. We also present the distribution of baryonic matter over these various objects. We show that light does not trace baryonic mass, since bright galaxies which contain most of the stars only form a small fraction of the mass associated with virialized and cooled halos. We consider two cosmologies: a critical density universe and an open universe. In both cases, our results agree with observations and show that hierarchical scenarios provide a good model for structure formation and can describe a wide range of objects which spans at least the seven orders of magnitude in mass for which data exist. More detailed observations, in particular of the clustering evolution of galaxies, will constrain the astrophysical models involved.
\end{abstract}

Key words. Cosmology: large-scale structure of Universe - galaxies: evolution - quasars: general quasars: absorption lines - intergalactic medium - galaxies: clusters

\section{Introduction}

In the standard cosmological scenario large-scale structures observed in the present universe were formed by the amplification through gravitational instability of small primordial density fluctuations (usually assumed to be Gaussian). Within hierarchical models (as in the CDM case: Peebles 1982) small scales collapse first to form bound objects which later merge to build more massive halos as larger scales become non-linear. This describes the evolution under the action of gravity of the dark matter component. However, observed objects (e.g., quasars, galaxies, Lyman- $\alpha$ clouds...) are formed by baryonic matter (gas, stars) which does not necessarily follow the behaviour of the dark matter component since it undergoes additional processes (e.g., radiative cooling). Moreover, by looking at specific astrophysical objects (e.g. bright galaxies) one selects peculiar dark matter environments where the former are most likely to arise. Thus, to relate observations to the underlying dark matter component (which is usually gravitationally dominant) and to test scenarios

Send offprint requests to: P. Valageas, e-mail: valag@spht.saclay.cea.fr for structure formation one needs to study the link between the dark and baryonic components. This can also constrain the astrophysical models used to build galaxies and other objects from the density field.

One tool to describe the distribution of objects in the universe, in addition to their abundance, is their twopoint correlation function which measures their clustering. Then one often defines the bias $b^{2}$ of these halos as the ratio of this quantity to the dark matter correlation function, which shows in a clear quantitative way whether these objects are more or less clustered than the dark matter component. In this article, we present the bias (and the correlation length) associated with various objects (galaxies, quasars, Lyman- $\alpha$ clouds, clusters). The formulation of this bias is obtained (Schaeffer 1985, 1987; Bernardeau \& Schaeffer 1992, 1999) within the framework of a general description of structure formation in the universe provided by a scaling model (Schaeffer 1984; Balian \& Schaeffer 1989) which describes the density field in the non-linear regime. These predictions specifically take into account the deep non-linearity of the density field and are conceptually different from those obtained using linear theory (e.g., Mo \& White 1996). Indeed, the 
latter authors evaluate the bias of primordial overdensities that will collapse later, using linear theory, with the implicit assumption that this bias is conserved during the course of evolution. They find the bias depends separately on the mass, radius and overdensity. Here, we use the bias calculated directly using non-linear theory. This bias is seen to depend on very specific internal properties of the considered non-linear objects, namely on a unique parameter $x$, quite close to the internal velocity dispersion within the objects, thus exhibiting a scaling law. Such a specific dependence has indeed been found to hold in numerical simulations (Munshi et al. 1999b), and we consider this approach to provide a more trustworthy description of the present clustering properties as well as their evolution at higher redshifts.

In the present paper, we specifically study the dependence of the bias on the observable properties of the considered objects (luminosity, column density) and its evolution with redshift. In contrast with most previous works, our analytical model relating dark to observable mass is not an ad-hoc parameterization. Indeed, it is simply a consequence of a general model (Valageas \& Schaeffer 1997) which has already been applied to galaxies (Valageas \& Schaeffer 1999), Lyman- $\alpha$ clouds (Valageas et al. 1999), clusters (Valageas \& Schaeffer 2000) and reionization studies (Valageas \& Silk 1999a,b). Thus, its predictions have already been compared with many observations (e.g., galaxy luminosity function, column density distribution of Lyman- $\alpha$ clouds, amplitude of the UV background radiation field, cluster X-ray luminosity function, ...). Hence there is no free parameter chosen in this article. Next, in addition to the bias we also present the distribution of baryonic matter over the various objects we describe.

This article is organized as follows. In Sect. 2 we describe our prescription for mass functions, while in Sect. 3 we recall how the bias is obtained in this model and we compare our formalism with other prescriptions. In Sect. 4 we present our results for the case of a critical density universe. We first describe the clustering of galaxies, quasars, Lyman- $\alpha$ clouds and clusters and then we study the repartition of baryonic matter within various classes of objects. Finally, in Sect. 5 we present the case of an open universe.

\section{Multiplicity functions}

Since we wish to describe the properties of various classes of objects like Lyman- $\alpha$ clouds and galaxies which are defined by specific constraints we need a formalism which can handle more general multiplicity functions than the usual mass function of "just-virialized halos". To this order, we shall assume that the non-linear density field obeys the scaling model detailed in Balian \& Schaeffer (1989). Thus, we define each class of objects (Lyman- $\alpha$ clouds or galaxies) by a constraint $\Delta(M, z)$ on the density contrast of the underlying dark-matter halo of mass $M$ at redshift $z$. The "just-virialized halos" correspond to the special case $\Delta(M, z)=\Delta_{\mathrm{c}}(z)$ with $\Delta_{\mathrm{c}} \sim 177$. For our purposes we consider the cases of a constant density contrast, arising from a virialization constraint, and of a constant radius (i.e. $(1+\Delta) \propto M)$, arising from a fixed cooling radius or Jeans length. In any case, we write the multiplicity function of these objects as (see Valageas \& Schaeffer 1997 for details):

$\eta(M, z) \frac{\mathrm{d} M}{M}=\frac{\bar{\rho}}{M} x^{2} H(x) \frac{\mathrm{d} x}{x}$.

Here, the parameter $x$ associated with a halo of mass $M$ at redshift $z$ is defined by:

$x(M, z)=\frac{1+\Delta(M, z)}{\bar{\xi}[R(M, z), z]}$,

where

$\bar{\xi}(R)=\int_{V} \frac{\mathrm{d}^{3} r_{1} \mathrm{~d}^{3} r_{2}}{V^{2}} \xi_{2}\left(\boldsymbol{r}_{1}, \boldsymbol{r}_{2}\right) \quad$ with $\quad V=\frac{4}{3} \pi R^{3}$

is the average of the two-body correlation function $\xi_{2}\left(\boldsymbol{r}_{1}, \boldsymbol{r}_{2}\right)$ over a spherical cell of radius $R$ and provides the measure of the density fluctuations in such a cell (thus typical objects have $x \sim 1$ ). The scaling function $H(x)$ depends on the initial power-spectrum of the density fluctuations and must be taken from numerical simulations. However, from theoretical considerations (Balian \& Schaeffer 1989) it is expected to satisfy the asymptotic behaviour:

$x \ll 1: H(x) \propto x^{\omega-2}, \quad x \gg 1: H(x) \propto x^{\omega_{\mathrm{s}}-1} \mathrm{e}^{-x / x_{*}}$

with $\omega \simeq 0.5, \omega_{\mathrm{s}} \sim-3 / 2, x_{*} \sim 10$ to 20 , and by definition it must satisfy

$\int_{0}^{\infty} x H(x) \mathrm{d} x=1$

These properties have been checked for various $P(k)$ by Bouchet et al. (1991), Colombi et al. (1992, 1994, 1995, 1997), Munshi et al. (1999a), while the mass functions obtained from (1) for various constraints $\Delta(M)$ (for a constant $\Delta$ which was taken from -0.5 to 5000 or for $(1+\Delta) \propto M)$ have been shown to provide reasonable approximations to numerical results in Valageas et al. (2000).

\section{Bias}

\subsection{Our formulation}

Within the framework of the scale-invariance of the manybody matter correlation functions $\xi_{p}\left(\boldsymbol{r}_{1}, \ldots, \boldsymbol{r}_{p}\right)$ which led to the mass function (1), one can show that in the highly non-linear regime the bias characteristic of two objects factorizes and is a function of the sole parameter $x$ introduced in Sect. 2, see Bernardeau \& Schaeffer (1992, 1999). Thus we write the correlation function of objects of type (1) with objects of type (2) as:

$\xi_{1,2}(r)=b\left(x_{1}\right) b\left(x_{2}\right) \xi(r)$

with

$x \ll 1: b(x) \propto x^{(1-\omega) / 2} \quad$ and $\quad x \gg 1: b(x) \propto x$. 
This behaviour has been successfully checked in numerical simulations by Munshi et al. (1999c). This allows us to obtain the redshift evolution of the bias associated with galaxies, Lyman- $\alpha$ clouds or quasars, once we define the constraints $\Delta(M, z)$ which characterize these various astrophysical objects (that in turn define the proper value of $x$ to be used).

\subsection{Comparison with some other models}

We can note that alternative models have been proposed in the litterature to describe the clustering of galaxies. Here we briefly compare our prescription to such models, in order to clarify their main differences. Thus, semianalytic models of galaxy and dark matter clustering based on Press-Schechter-like ideas have recently been proposed (Seljak 2000; Peacock \& Smith 2000). They describe the dark matter density field as a collection of smooth halos with a mean density profile. The mass function of these "just-virialized" halos (defined by an overall density contrast $\left.\Delta=\Delta_{c} \sim 177\right)$ is obtained from a slightly modified Press-Schechter mass function (Press \& Schechter 1974). By construction, such a model neglects the substructures of virialized halos since they are described by an average density profile. This is not a problem at large scales where the correlations among galaxies are governed by the distribution of their host virialized halos (note that since the Press-Schechter prescription is based on the linear density field these correlations should actually apply to very large scales which are still in the linear regime). However, at scales of the order of the size of large "just-virialized" halos the clustering of galaxies is set by the distribution of these galaxies within those larger objects (which actually correspond to clusters of galaxies at large masses).

Hence one needs to add a specific prescription to cope with this regime. Thus, one introduces the mean number $\langle N\rangle(M)$ of galaxies contained in halos of mass $M$. This function is derived from $N$-body simulations or observations. Then, one puts a galaxy at the center of the halo and the $(N-1)$ remaining galaxies at random within the halo, following the mean dark matter density profile. In order to include the fluctuations of $N(M)$ one can even reproduce the second moment $\langle N(N-1)\rangle(M)$ obtained from simulations (Seljak 2000). However, it is clear that the predictive power of such a model is quite limited. Indeed, the output of the model (i.e. the two-point correlation function of galaxies) is almost directly related to the input (the first two moments of the galaxy distribution within virialized halos). Thus, this can at best serve as a test of $\mathrm{N}$-body simulations if this data is taken from simulations (while it would not make much sense to obtain this data from the observations one would later on try to recover). In particular, in order to derive the $p$-point correlation function of galaxies in this line one would need the moment $\left\langle N^{p}\right\rangle$ of order $p \ldots$ On the other hand, if one only includes the mean $\langle N\rangle(M)$ (taken from observations for instance) as input, one can argue that the model tests whether the spatial galaxy distribution is well described by the mean dark matter density profile. However, as shown in Valageas (1999) such a model contradicts the results of numerical simulations for the dark matter distribution itself when one takes into account the constraints provided by the first five order moments of counts-in-cells statistics. This implies that one could not get higher-order moments (e.g., the three-point galaxy correlation function) from this model.

Moreover, it is not clear how much information one can draw from the mere comparison of the galaxy powerspectrum predicted by the model with observations. Indeed, any sensible model should provide a reasonable agreement with the data since it should more or less follow the dark matter power-spectrum which is itself normalized to observations of large-scale clustering (through $\sigma_{8}$ ). Then, it seems difficult to obtain narrow constraints on the input parameters from only one curve, in view of the number of these free parameters and the approximations involved in such models (e.g., importance of the scatter of galaxy properties, shape of the distribution $P(N)$ within virialized halos, role of dark matter substructures, ...).

Such approaches differ from ours in many respects. First, we use a mass function derived from a scaling ansatz for the dark matter density field (Balian \& Schaeffer 1989) which applies to the non-linear regime. It is fully specified by the sole function $H(x)$, obtained from counts-incells statistics, and by the behaviour of the non-linear power-spectrum. Most importantly, it allows us to get various multiplicity functions for different classes of objects in addition to "just-virialized" halos, defined by a relation $\Delta(M)$ or $R(M)$ which specifies their overall density contrast or radius as a function of their mass (Valageas \& Schaeffer 1997; Valageas et al. 2000). This enables us to bypass the link between galaxies and "justvirialized" halos which hinders the approaches described above. Indeed, we directly recognize the galaxies themselves, defined by a relation $R(M)$ obtained from cooling constraints (Valageas \& Schaeffer 1999, 2000).

Then, as explained above the clustering of these objects is obtained (Bernardeau \& Schaeffer 1992, 1999) from the behaviour of the $p$-point correlation functions $\xi_{p}$ which led to the mass function (1). Hence it does not require any additional information. Moreover, although we restrict ourselves to the two-point correlation functions, our formulation also predicts the $p$-point correlation functions of any order $p$ for all the objects we study here (galaxies, QSOs, ...). This clearly illustrates the predictive power of our model. Besides, although we do not take into account the scatter of galaxy properties with respect to their mass, our procedure automatically includes the part of the scatter of the galaxy distribution with respect to the distribution of "just-virialized" halos which is due to the statistics of the dark matter itself.

Finally, the spirit of our present study is rather different from the works which are mainly intended to describe galaxy clustering (Seljak 2000; Peacock \& Smith 2000) as 
we merely compare with observations the predictions of a general model of structure formation which was fully constrained in previous papers which dealt with its consequences for galaxies, QSOs, Lyman- $\alpha$ clouds and clusters. In particular, we have no free parameters left to improve the agreement with the observed galaxy clustering and we check our model against a vast array of observations, ranging from Lyman- $\alpha$ clouds up to clusters, through galaxies and QSOs. Thus, our goal is not to use observations of galaxy clustering to constrain some free parameters, but to check whether a sensible scenario of structure formation can match this data. Besides, since we use an analytic model where the various physical processes can be clearly identified, we can get some valuable insight into the clustering properties of the range of objects we study here. For instance, we shall see that a model like ours can explain in simple physical terms the qualitative and quantitative differences between the redshift evolution of the clustering of bright galaxies and faint galaxies, small Lyman $-\alpha$ forest clouds and damped systems, or QSOs and galaxies.

Another method to study the clustering of galaxies is to use $N$-body simulations, which may be supplemented by semi-analytic modelling of star-formation or galaxy formation (e.g., Benson et al. 2000a). In principle, this allows one to avoid the assumptions and approximations involved in an analytic model like ours to describe the dark matter density field. However, this technique may be limited by numerical resolution and computer time constraints. In any case, this is certainly a promising method but it does not provide yet the clustering properties of the full range of objects we study here in a unified fashion.

\section{Critical density universe}

We first consider the case of a critical density universe $\Omega_{\mathrm{m}}=1$ with a CDM power-spectrum (Davis et al. 1985), normalized to $\sigma_{8}=0.5$. We choose a baryonic density parameter $\Omega_{\mathrm{b}}=0.04$ and $H_{0}=60 \mathrm{~km} \mathrm{~s}^{-1} / \mathrm{Mpc}$. We use the scaling function obtained by Bouchet et al. (1991) for $H(x)$.

\subsection{Galaxies}

\subsubsection{Present universe}

We use the model of galaxy formation and evolution described in detail in Valageas \& Schaeffer (1999), where it was checked against many observations (luminosity function, Tully-Fisher relation...). We define galaxies by two constraints: 1) a virialization condition $\Delta>\Delta_{\mathrm{c}}$ where $\Delta_{\mathrm{c}} \sim 177$ is given by the usual spherical model, and 2) a cooling constraint $t_{\text {cool }}<t_{\mathrm{H}}$ which requires the gas to cool within a few Hubble times at formation. At high virial temperature and low redshift, the condition 2) is the most stringent and can be approximated by a constant radius constraint $R=R_{\text {cool }}$. At high redshift it becomes irrelevant since any halo which satisfies 1 ) also satisfies 2 ). These two conditions define for galaxies the constraint
$\Delta(M, z)$ we introduced in Sect. 2. Once we have identified in this manner the dark-matter halos which correspond to galaxies we still need to set up a model for star formation to obtain the stellar properties of these objects. As detailed in Valageas \& Schaeffer (1999) our model involves 4 components: (1) short-lived stars which are recycled, (2) long-lived stars which are not recycled, (3) a central gaseous component which is deplenished by star formation and ejection by supernovae winds, replenished by infall from (4), a diffuse gaseous component. This allows us to obtain the luminosity of these galaxies. Since observations usually refer to the mean bias (or correlation function) of galaxies above a given luminosity, we define:

$b(>L)=\frac{\int_{L}^{\infty} b(L) \eta_{\mathrm{g}}(L) \frac{\mathrm{d} L}{L}}{\int_{L}^{\infty} \eta_{\mathrm{g}}(L) \frac{\mathrm{d} L}{L}}$,

where we used the factorization of the bias, see (4). We present in Fig. 1 the dependence of the bias on the $B$-band luminosity.

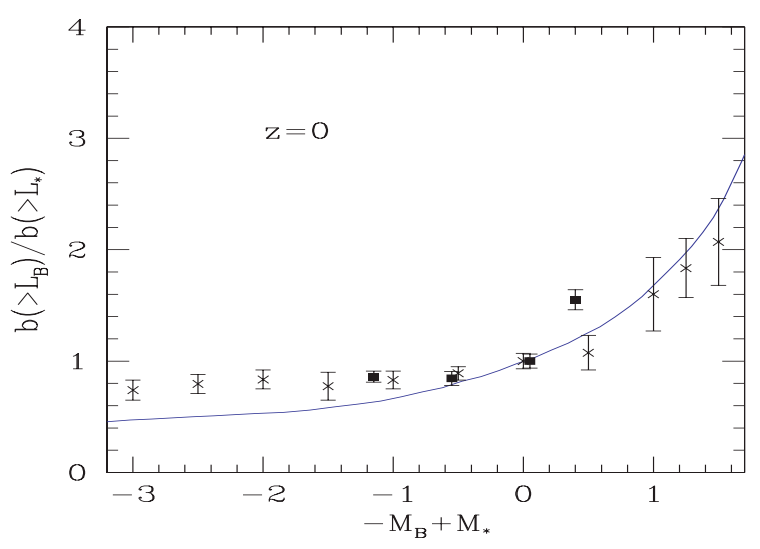

Fig. 1. The luminosity dependence of the bias $b\left(>L_{B}\right)$ at $z=$ 0 . The solid line is the mean bias $b\left(>L_{B}\right) / b\left(>L_{*}\right)$ of galaxies brighter than $L_{B}$ normalized to the bias of galaxies brighter than $M_{*}=-19.5$. The data points are from Benoist et al. (1996) (crosses) and Willmer et al. (1998) (squares)

We see that we recover the trend of larger bias for brighter galaxies, although our dependence is somewhat steeper than the observed relation. However, we note that in our analytical model each luminosity is associated with a specific object of a given mass, radius, ... In a more realistic model one should include some scatter in this relation which would lessen the final luminosity dependence of the bias (for a large scatter such that the luminosity is uncorrelated with the properties of the underlying dark matter halo we would have no $B$-magnitude dependence left).

From the relation (4) we can also obtain the crosscorrelation of galaxies of luminosity $L_{B}$ with all galaxies, in a fashion similar to (6). We can convert the bias $b$ evaluated in this manner into a correlation length $r_{0}(b)$ defined by:

$r_{0}(b)=b^{2 / \gamma} r_{0}$ 
where $\gamma=1.8$ is the approximate slope of the correlation function and $r_{0}$ is the correlation length of the dark matter density field. Indeed in our model the correlation functions of various objects and of the matter distribution all have the same slope in the non-linear regime (note that Dave et al. 1999 find from numerical simulations that the bias shows very little scale dependence, if any, in the nonlinear regime). This prescription allows us to compare in Fig. 2 our results to observations performed by Loveday et al. (1995).

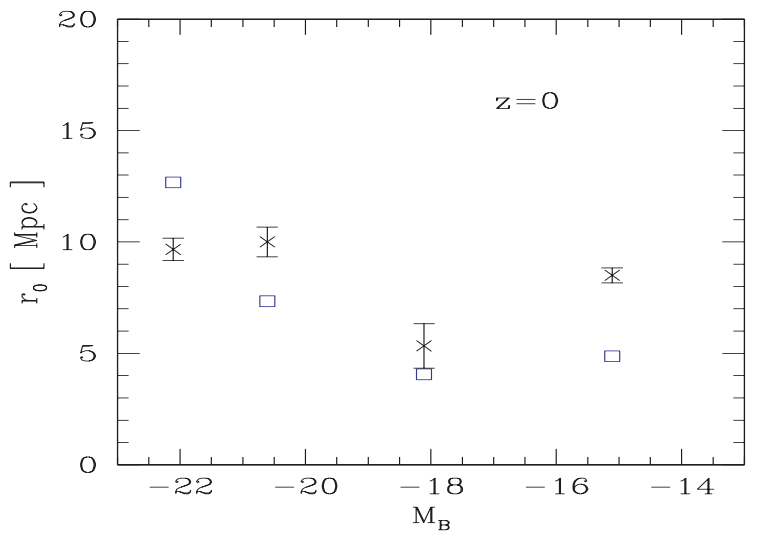

Fig. 2. From left to right the correlation length $r_{0}$ at $z=0$ of the correlation function of galaxies between the magnitude bins $-22.8<M_{B}<-20.8,-20.8<M_{B}<-19.8$ and $-19.8<$ $M_{B}<-15.8$ with all galaxies (i.e. with $-22.8<M_{B}<-15.8$ ). The point on the far right is the correlation length of all galaxies with $-22.8<M_{B}<-15.8$. The data points (crosses) are from Loveday et al. (1995) and the squares are our predictions

We see that our results are compatible with the observations. We find an increase of the bias (hence of $r_{0}$ ) for brighter galaxies in accordance with the data, except for the last point at $-22.8<M_{B}<-20.8$ where the bias is measured to be slightly smaller than for $-20.8<M_{B}<$ -19.8. Nevertheless, since this contradicts the trend observed by Benoist et al. (1996) this disagreement is probably not conclusive.

We present in Fig. 3 the correlation function of galaxies brighter than $M_{B}=-18.6$ at $z=0$. Our results agree reasonably well with observations, although the slope we obtain may be a bit too steep at large scales. This is a well-known discrepancy for CDM initial conditions with $\Omega_{\mathrm{m}}=1$ and could be improved with a slightly different initial power-spectrum. The dashed line is the correlation function given by the linear theory multiplied by the relevant bias factor. Hence it coincides with the actual correlation function at large scales. At small scales the non-linear dynamics increases the clustering as compared to the linear predictions, in agreement with observation. Note that in principle the non-linear predictions are valid for $\bar{\xi} \gg 1$, but are in practice seen to hold as soon as $\bar{\xi} \geq 1$.

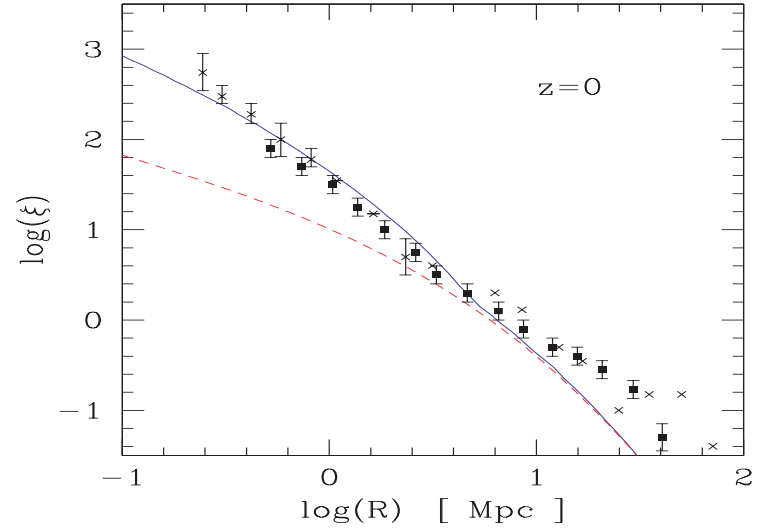

Fig. 3. The correlation function of galaxies brighter than $M_{B}=-18.6$ at $z=0$ (solid line). The dashed line is the linear prediction. The data points are from Baugh (1996) (squares) for $-21.6<M_{B}<-18.6$ and from Loveday et al. (1995) (crosses) for $-22.8<M_{B}<-15.8$

\subsubsection{Redshift evolution}

We now turn to our predictions for the redshift evolution of the bias associated with galaxies recognized by their $B$-band luminosity, shown in Fig. 4.

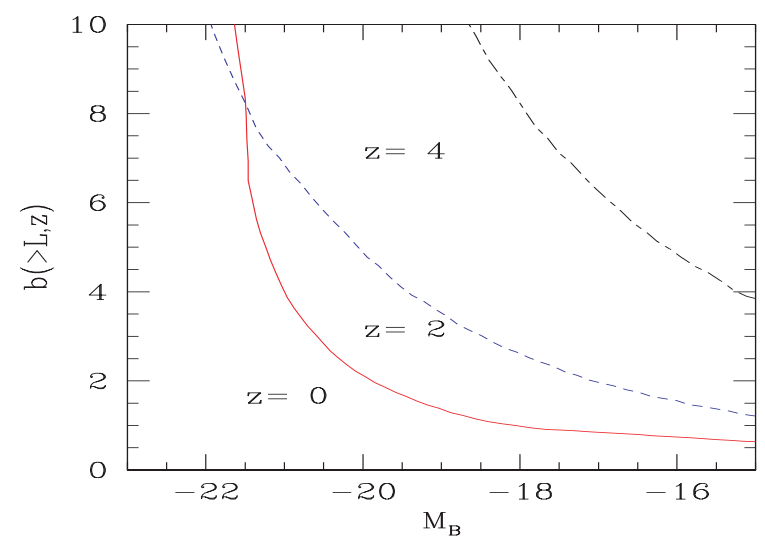

Fig. 4. The redshift evolution of the relation luminosity-bias. The solid line corresponds to $z=0$, the dashed line to $z=2$ and the dot-dashed line to $z=4$

At "low" luminosities $M_{B}>-21$, the bias grows with redshift since galactic halos correspond to increasingly rare overdense regions (because structure formation was not as developed as it is today). For very bright galaxies $M_{B}<-21$ we recover the same trend except at low redshifts where the bias suddenly increases with time. This evolution is due to a luminosity effect. Indeed, in our model at low $z<1$ very massive galaxies see their luminosity decrease with time as their initial gas content gets exhausted by star formation (which is very efficient in this high-density environment). As a consequence, when selecting galaxies by a fixed $B$-band luminosity, one looks at more massive, higher virial temperature halos at $z=0$ than at $z=1$. This shift to higher density fluctuations acts opposite to the usual trend seen for lower luminosity 
galaxies and leads to the increase with time of the bias at low $z$ for these very bright magnitudes.

We present directly in Fig. 5 the redshift evolution of the bias associated with galaxies brighter than three $B$-band magnitude thresholds. This clearly shows the dependence on luminosity of the redshift evolution itself.

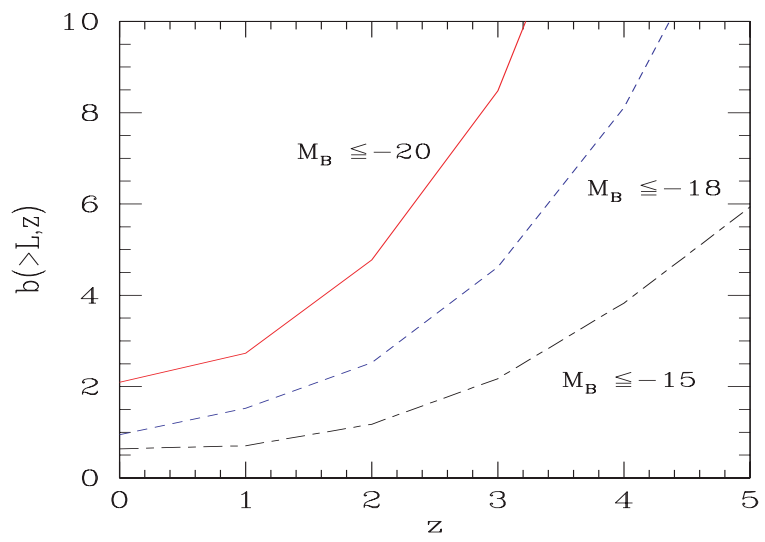

Fig. 5. The redshift evolution of the bias associated with galaxies below various $B$-band magnitude thresholds. The solid line corresponds to $M_{B} \leq-20$, the dashed line to $M_{B} \leq-18$ and the dot-dashed line to $M_{B} \leq-15$

We show in Fig. 6 the redshift evolution of the comoving correlation length $r_{0}$ associated with galaxies brighter than various magnitude thresholds. The two data points at $z<0.1$ correspond to $z=0$ but they have been slightly shifted to be more clearly seen. We can see that our results agree reasonably well with observations, except for the data points from Le Fevre et al. (1996) which however are inconsistent with data from Carlberg et al. (1998) and Postman et al. (1998). The comoving number density of Lyman-break galaxies at $z=3$ is $n \gtrsim 2.710^{-3} \mathrm{Mpc}^{-3}$ (see Adelberger et al. 1998) which is also the abundance of galaxies brighter than $M_{B}=-18.7$ (and $M \geq 510^{11} M_{\odot}$ ) in our model. The square at $z=3$ shows our prediction for the clustering strength of galaxies with this abundance. We see that this simple identification leads to good agreement with observations (filled triangle). The comoving correlation length of galaxies first decreases at higher $z$, following the decline of the dark-matter two-point correlation function. This trend is even larger for very bright galaxies since it is amplified by the decline of their bias, as shown in Fig. 5. However, at high redshifts $z>2, r_{0}$ increases with $z$ because of the strong growth of the bias. Of course, this trend is stronger for brighter galaxies. We note that contrary to Kauffmann et al. (1999) we obtain a "dip" in the evolution of $r_{0}$, while these authors only found this feature for a low-density flat cosmology. This is explained by the slower growth with $z$ of the bias associated with galaxies in our model, as compared to theirs, which is insufficient to override at low $z$ the decrease of clustering measured by the decline of the dark-matter correlation function. Thus, such a feature depends on the astrophysical model used to build galaxies. However,

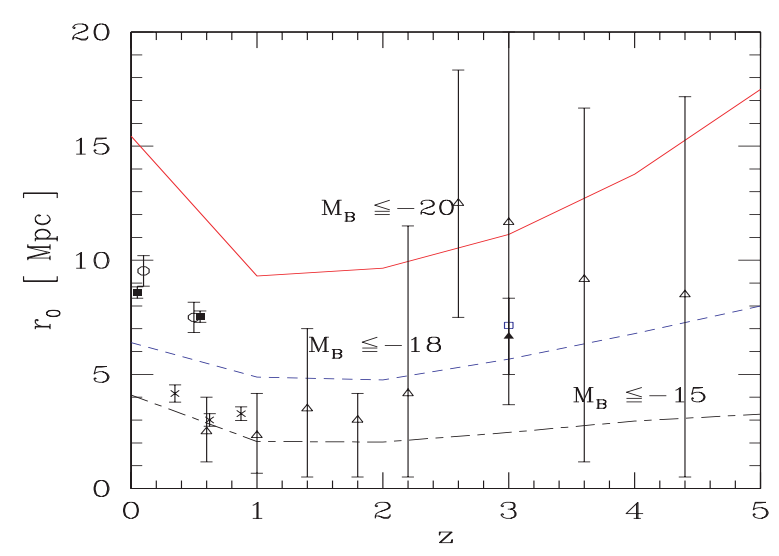

Fig. 6. The redshift evolution of the correlation length $r_{0}$ (in comoving $\mathrm{Mpc}$ ) associated with galaxies below various $B$-band magnitude thresholds. The solid line corresponds to $M_{B} \leq-20$, the dashed line to $M_{B} \leq-18$ and the dot-dashed line to $M_{B} \leq-15$. The data points are from Le Fevre et al. (1996) (crosses, $-21.3<M_{B}<-18.3$ ), Carlberg et al. (1998) (filled squares, $M_{B}<-19.5$ ), Postman et al. (1998) (circles, $I<20$ ) and Magliocchetti \& Maddox (1999) (open triangles). The filled triangle at $z=3$ corresponds to Lyman-break galaxies (observations from Adelberger et al. 1998) while the open square is our prediction for galaxies which have the same abundance as Lyman-break galaxies

we note that observations show that $r_{0}$ decreases with larger $z$ in the range $0 \leq z \leq 0.5$, in agreement with our model. We recall here that our results are direct consequences of a detailed model for structure formation which has already been compared with many observations, so that no attempt was made to "improve" the agreement of our predictions with the data. In fact, as shown by the discrepancy between various observations, the latter still have a significant uncertainty. However, we recover the general trend of a decrease of $r_{0}$ up to $z \sim 2$ and a subsequent growth at higher redshifts. This behaviour also agrees with the results obtained by Baugh et al. (1999) from a semi-analytic model.

\subsection{Quasars}

From the description of galaxies presented above we have also developed a model for the quasar luminosity function, as detailed in Valageas \& Silk (1999a). We assume that a fraction $\lambda_{\mathrm{Q}} \leq 1$ of galactic halos host a quasar with a mass $M_{\mathrm{Q}}$ proportional to the mass of gas $M_{\mathrm{gc}}$ available in the inner parts of the galaxy: $M_{\mathrm{Q}}=F M_{\mathrm{gc}}$. For most galaxies this also implies $M_{\mathrm{Q}} \simeq F M_{\mathrm{S}}$ where $M_{\mathrm{S}}$ is the stellar mass and we use $F=0.01$. We also assume that quasars shine at the Eddington limit with a radiative efficiency $\epsilon=0.1$, which means that the quasar life-time is $t_{\mathrm{Q}}=4.4 \epsilon 10^{8} \mathrm{yr}$. This determines the quasar bolometric luminosity:

$L_{\mathrm{Q}}=\frac{\epsilon M_{\mathrm{Q}} c^{2}}{t_{\mathrm{Q}}}$. 
We present in Fig. 7 the redshift evolution of the quasar luminosity - bias relation. Here $b\left(>L_{B}, z\right)$ is the bias of quasars brighter than $L_{B}$ at redshift $z$.

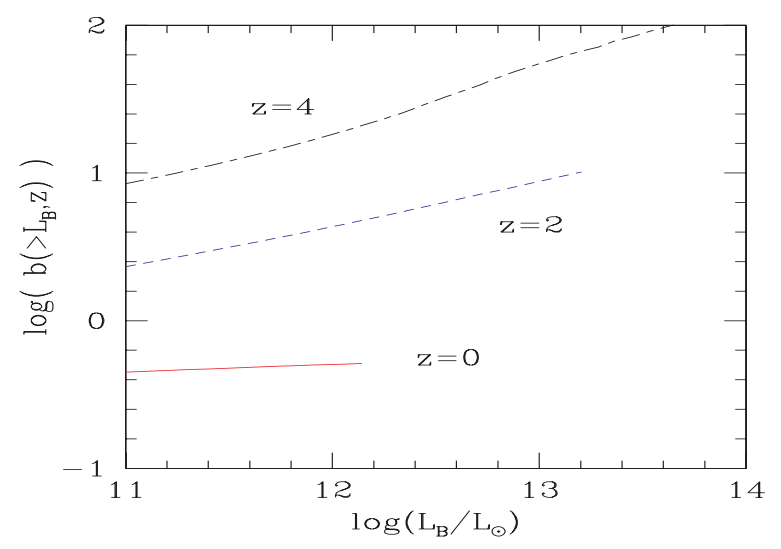

Fig. 7. The redshift evolution of the bias associated with quasars above various luminosity thresholds. The solid line corresponds to $z=0$, the dashed line to $z=2$ and the dot-dashed line to $z=4$

We see that the bias increases with redshift since at higher $z$ quasars of a given luminosity correspond to rarer density fluctuations. The high luminosity cutoff seen in the figure comes from the fact that in our model very massive galaxies have already consumed most of their gas content at low $z$, as explained in the previous section, so that the maximum quasar luminosity declines with time because of fuel exhaustion. Of course, at higher $z$ the quasar luminosity function cutoff also declines because of the knee of the mass function of collapsed objects (Valageas \& Silk 1999a).

From the bias $b\left(>L_{B}, z\right)$ we can derive the comoving correlation length of quasars. This is shown in Fig. 8. We can check that our results agree reasonably well with observations. In particular, we recover the increase with redshift of the correlation length. La Franca et al. (1998) noticed that this behaviour differs from the decrease of $r_{0}$ observed for galaxies from $z=0$ to $z=1$ which we also obtain, as shown in Fig. 6. Thus a simple hierarchical model like ours is able to simultaneously explain both of these redshift dependences. The stronger increase with redshift of the correlation length associated with quasars, as compared with galaxies, also translates into the faster growth of their bias factor, see Figs. 4 and 7. This difference between galaxies and quasars is due to the redshift evolution of their respective ratios of (mass)/(luminosity). As described above, in our model the luminosity of a quasar is proportional to its mass of gas, see (8), and to the mass of stars in the host galaxy. Hence the bias of quasars of a given luminosity grows strongly with redshift since objects with a fixed mass of central gas are increasingly rare at earlier times when structure formation is less advanced. This overrides the decrease of the dark matter correlation length with $z$ and it leads to a rise of $r_{0}\left(>L_{B}\right)$,

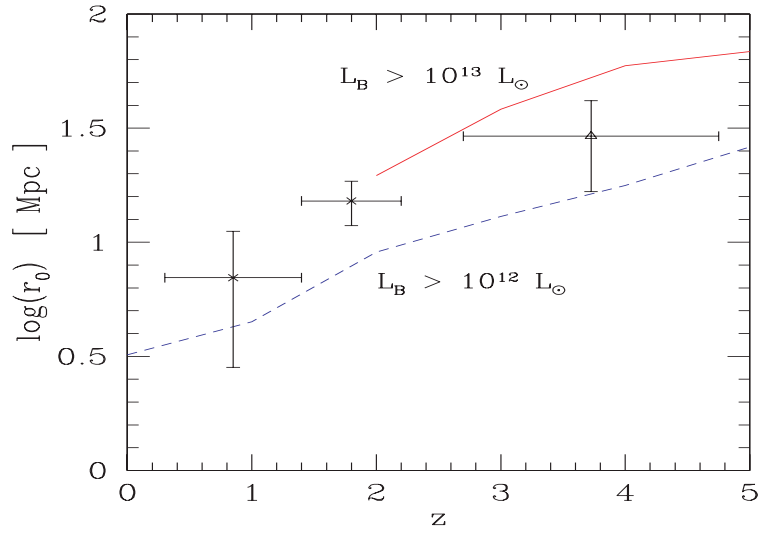

Fig. 8. The redshift evolution of the comoving correlation length associated with quasars above various luminosity thresholds. The dashed line corresponds to $L_{B}>10^{12} L_{\odot}$ and the solid line to $L_{B}>10^{13} L_{\odot}$. The two crosses are observations from La Franca et al. (1998) for $0.3<z<1.4$ (lower point) and $1.4<z<2.2$ (upper point). The triangle shows the result at $2.7<z<4.75$ of observations by Stephens et al. (1997)

as seen in Fig. 8. On the other hand, galaxies with a constant mass of star-forming gas had a higher luminosity in the past because the ratio $M_{\mathrm{S}} / L$ decreases with redshift (see for instance Fig. 17 in Valageas \& Schaeffer 1999). This is due to the fact that the smaller age of the universe $t_{\mathrm{H}}$ selects more massive and brighter stars with a life-time $t_{*} \propto t_{\mathrm{H}}$. In other words, at later times the contribution from faint long-lived stars which progressively accumulated in the galaxy ever since it was born becomes increasingly important as compared with the contribution of bright newly-born short-lived stars which quickly disappear as supernovae and thus cannot accumulate. This implies that by looking at a fixed luminosity one selects a smaller mass of gas and stars at higher redshift.

Thus, if we start at $z=0$ with galaxy and quasar luminosities which correspond to the same host halos, at large redshift $z>0$ the galaxies with the same luminosity correspond to smaller halos than the quasar hosts. Hence galaxies correspond to less extreme objects and have a smaller bias. Thus at low $z$ their correlation length follows the decreases of the dark matter $r_{0}$, until $z \sim 1$, beyond which the rise of their bias becomes sufficiently strong to lead to a growth of $r_{0}\left(>L_{B}\right)$, see Fig. 6 . The precise amplitude of these redshift evolutions depends somewhat on the astrophysical models used for quasars and stars $(\mathrm{IMF}, \ldots)$. However, the agreement we obtain with these observations is remarkable since our model was built independently of these considerations, with no free parameter left for this present discussion. Thus this behaviour appears as a natural outcome of such models which suggests they provide a realistic description of the global properties of star and quasar formation, even though the details of these processes are still poorly known. 


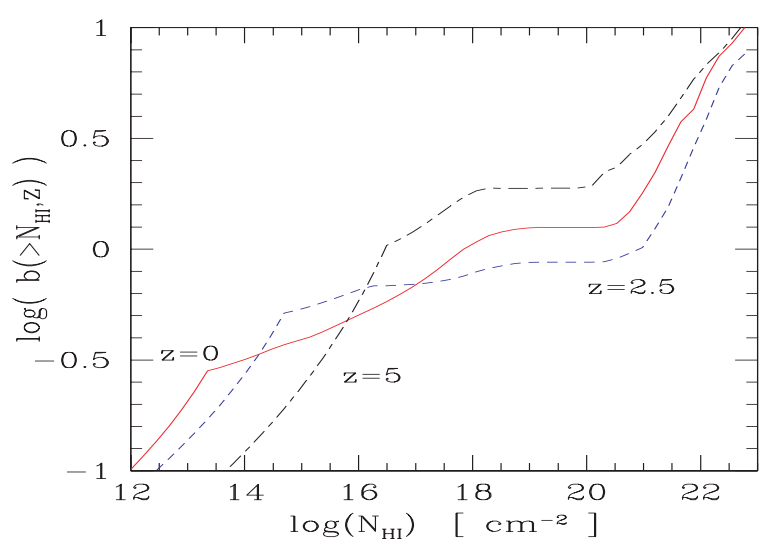

Fig. 9. The redshift evolution of the bias associated with Lyman- $\alpha$ absorbers above various column density thresholds. The solid line corresponds to $z=0$, the dashed line to $z=2.5$ and the dot-dashed line to $z=5$

\subsection{Lyman- $\alpha$ absorbers}

As shown in Valageas et al. (1999), the formalism introduced in Sect. 2 also allows us to build a model for Lyman$\alpha$ clouds, some of which are objects with a lower density than the mean density of the universe. We describe these absorbers as three different populations of objects. Low density halos with a small virial temperature see their baryonic density fluctuations erased over scales of the order of the Jeans length $R_{\mathrm{J}}$. These mass condensations form our first class of objects, defined by the scale $R_{\mathrm{J}}$, which can be identified with the Lyman- $\alpha$ forest. They correspond to low density clouds which can even be underdense (but stick out within very low-density regions or voids). On the other hand, potential wells with a large virial temperature maintain their density profile. Thus, they constitute a second population of absorbers where the column density observed along a line of sight depends strongly on the impact parameter. They can be identified with the Lyman-limit systems. Finally, the deep neutral cores of these halos (because of self-shielding) form our third class of objects which correspond to the damped systems.

We present in Fig. 9 the redshift evolution of the bias associated with Lyman- $\alpha$ absorbers above various column density thresholds.

We note that large column density absorbers have a bias greater than unity since they correspond to high density fluctuations (massive halos) while forest clouds which are low density fluctuations located in underdense areas have a small bias $b<1$. For $N_{\mathrm{HI}}<10^{14} \mathrm{~cm}^{-2}$ the bias decreases at larger redshift because the higher mean density of the universe implies that to observe a constant small column density one must look at increasingly underdense objects. In a similar fashion, for Lyman-limit or damped systems the bias first declines from $z=0$ to $z=2.5$. However, since these objects correspond to virialized halos the bias eventually grows with $z$ as the structure formation process is less advanced. Of course there is also an intermediate range of $N_{\mathrm{HI}}$ where the behaviour is more intricate. We display the redshift evolution of the bias

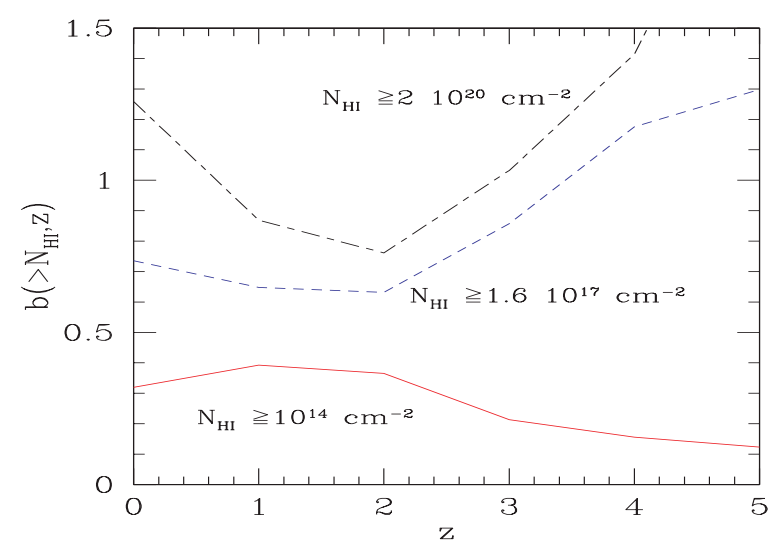

Fig. 10. The redshift evolution of the bias associated with Lyman- $\alpha$ absorbers above various column density thresholds. The solid line corresponds to $N_{\mathrm{HI}} \geq 10^{14} \mathrm{~cm}^{-2}$, the dashed line to $N_{\mathrm{HI}} \geq 1.610^{17} \mathrm{~cm}^{-2}$ and the dot-dashed line to $N_{\mathrm{HI}} \geq 210^{20} \mathrm{~cm}^{-2}$

associated with clouds above three different column densities thresholds in Fig. 10.

We see that the redshift evolution of the bias of Lyman$\alpha$ absorbers can display a non-trivial behaviour, since many processes play a role in the properties of these clouds, including the amplitude of the UV background radiation field. Thus, a phenomenological parameterisation of the form $b \propto N_{\mathrm{HI}}^{\alpha}(1+z)^{\beta}$ is unlikely to provide an accurate description. Note however that intermediate clouds in the range $10^{16}<N_{\mathrm{HI}}<10^{21} \mathrm{~cm}^{-2}$ have a bias which remains reasonably close to unity at all redshifts $z<5$ and thus can be used as tracers of the matter density field. A comparison of our results with observations for the two-point correlation function in velocity space is described in Valageas et al. (1999).

\subsection{Clusters}

Finally, we show in Fig. 11 the redshift evolution of the correlation length associated with clusters above various virial temperature thresholds. Here we simply defined clusters as "just-virialized" halos characterized by the usual density contrast $\Delta_{\mathrm{c}} \sim 177$. A detailed description of our model for clusters and the evolution of their X-ray luminosity function is presented in Valageas \& Schaeffer (2000). We see that our results agree reasonably well with observations. As was the case for quasars, the rise with redshift of the bias associated to these halos leads to an increase of their correlation length. However, this increase is slower than for quasars, see Fig. 8, because here we select these halos by a constant temperature threshold. This implies that their mass evolves as $M \propto(1+z)^{-3 / 2}$ hence we consider smaller halos at high $z$ which are more common (and have a smaller bias) than objects of constant mass. Thus, we have obtained a simple model of structure formation which is consistent with observations from Lyman- $\alpha$ clouds $\left(M \sim 10^{8} M_{\odot}\right)$ up to clusters $\left(M \sim 10^{15} M_{\odot}\right)$, i.e. over 7 orders of magnitude. 


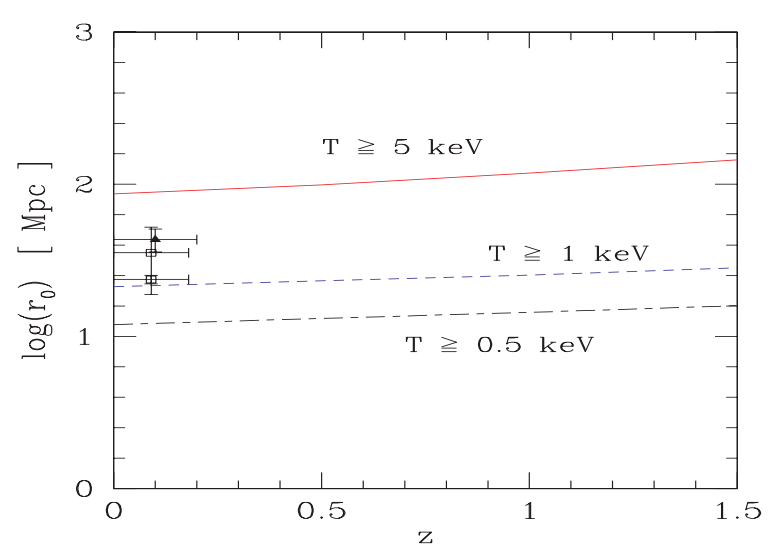

Fig. 11. The redshift evolution of the comoving correlation length associated with clusters above various virial temperature thresholds. The dot-dash line corresponds to $T \geq 0.5 \mathrm{keV}$, the dashed line to $T \geq 1 \mathrm{keV}$ and the solid line to $T \geq 5 \mathrm{keV}$. The two squares are observations from Croft et al. (1997) at $z<0.18$ for clusters with a richness $\mathcal{R} \geq 50$ (lower point) and $\mathcal{R} \geq 110$ (upper point). The triangle shows the result at $z<0.2$ of observations by Borgani et al. (1999) for the X-ray brightest Abell cluster sample

\subsection{Distribution of matter}

The models we have built in previous studies for galaxies (Valageas \& Schaeffer 1999) and Lyman- $\alpha$ clouds (Valageas et al. 1999) allow us to obtain the redshift evolution of the fraction of baryonic matter associated with various components.

We present in Fig. 12 the contributions of stars, galactic gas and Lyman- $\alpha$ forest clouds. In our model the sum of these three constituents is unity. Indeed, all the matter is embedded within mass condensations which we identify with galaxies (deep potential wells where the gas can cool) or with Lyman- $\alpha$ forest clouds (small density fluctuations heated by the UV background radiation). We see that the mass fraction within stars is always small $(\sim 10 \%$ at $z=0$ ) while the contribution of Lyman- $\alpha$ forest clouds shows a fast increase with redshift to constitute $\sim 70 \%$ of the matter at $z=5$ when there are few deep potential wells. Bright galaxies $\left(M_{B}<-19.5\right)$ contain most of the mass embedded within stars but they only constitute a small part of the gas which was able to cool. Moreover, their total mass of gas starts declining at low redshifts $z<1$ as it is converted into stars. This trend does not appear for faint galaxies because their star formation process is less efficient while they accrete gas which was previously part of the Lyman- $\alpha$ forest clouds. Moreover, some of these small galaxies have just formed as new potential wells have built up. Note that if the IGM is reheated by supernovae or quasars up to $T \sim 510^{5} \mathrm{~K}$, which is suggested by the observed relation temperature - X-ray luminosity of clusters (e.g., Ponman et al. 1999), the fraction of matter which has been able to cool and appears as galactic gas in Fig. 12 would be smaller. This was studied in details in Valageas \& Silk (1999b).

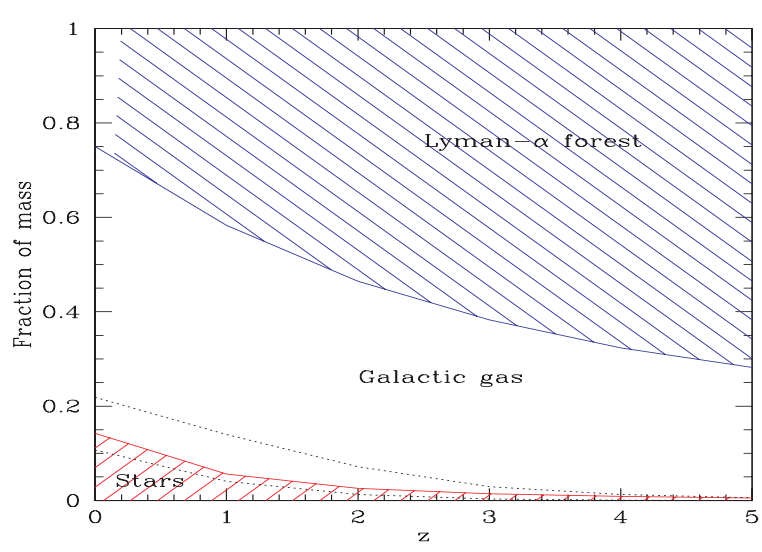

Fig. 12. The redshift evolution of the fraction of baryonic mass embedded within Lyman- $\alpha$ forest clouds (upper shaded area), galactic halos (white area) and stars (lower shaded area). The dotted lines separate the contributions from bright $\left(M_{B}<-19.5\right.$, lower part) and faint $\left(M_{B}>-19.5\right.$, upper part $)$ galaxies for stars and gas, respectively

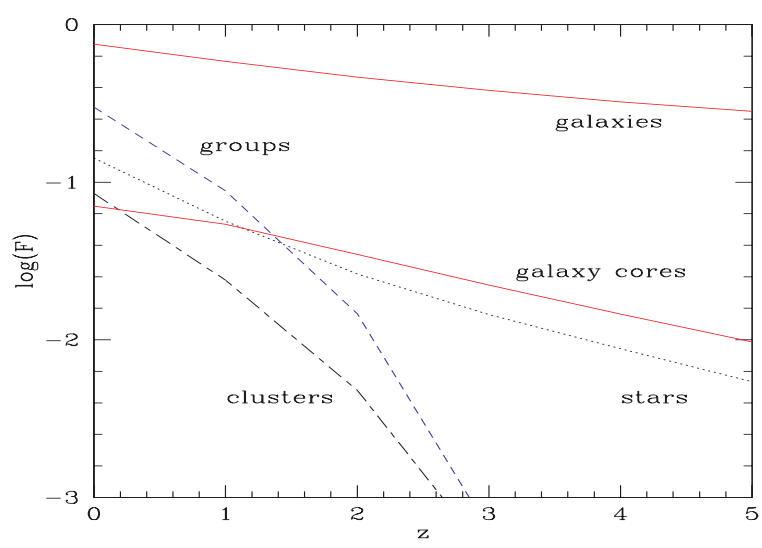

Fig. 13. The redshift evolution of the fraction $F$ of baryonic mass embedded within galaxies (upper solid line: total mass, lower solid line: gas in the core), groups (dashed line), clusters (dot-dashed line) and stars (dotted line)

We show in Fig. 13 the mass fraction we associate with groups and clusters of galaxies, as well as with stars and galaxies. We define groups as virialized halos (i.e. with a density contrast $\Delta_{\mathrm{c}}$ ) which do not satisfy the constraint $t_{\mathrm{cool}}<t_{\mathrm{H}}$. They correspond to massive potential wells with a large virial temperature (at high $T$ we have $t_{\text {cool }} \sim \sqrt{T}$ ). At high redshifts this contribution declines since an increasing proportion of virialized halos can cool (indeed $t_{\text {cool }} \propto 1 / \rho$ while $t_{\mathrm{H}} \propto 1 / \sqrt{\rho}$ ), see also Valageas $\&$ Schaeffer (1999). We identify clusters as groups with a virial temperature $T \geq 1 \mathrm{keV}$. At low $z$ they only form a small sub-class of galaxy groups while at high $z$ all groups have large virial temperatures $T \geq 1 \mathrm{keV}$ as explained above. Note that in our model the mass associated with groups is also associated to the member galaxies, so that it is a part of the fraction of matter recognized as embedded within galaxies, shown in Figs. 12 and 13 (upper solid line). A detailed study of groups and clusters in presented in Valageas \& Schaeffer (2000). 
Table 1. Baryonic parameter $\Omega_{\mathrm{i}}$ associated with various objects at $z=0$ and $z=3$, for a critical universe with $\Omega_{\mathrm{b}}=0.04$. The second line which appears for some contributions is the observational estimate from Fukugita et al. (1998)

\begin{tabular}{ccc}
\hline & $z=0$ & $z=3$ \\
\hline \hline stars & 0.0057 & 0.0006 \\
& $0.002-0.0057$ & \\
\hline galactic gas & 0.024 & 0.015 \\
\hline groups & 0.012 & 0.00002 \\
& $0.011-0.045$ & \\
\hline clusters & 0.0034 & 0.00002 \\
& $0.0014-0.0044$ & \\
\hline Lyman- $\alpha$ forest & 0.01 & 0.025 \\
& & $0.01-0.05$ \\
\hline
\end{tabular}

From the fraction of matter $F_{\mathrm{i}}$ associated with various objects and the baryonic density parameter $\Omega_{\mathrm{b}}=0.04$ we used in our model, we also obtain the baryonic density parameters $\Omega_{\mathrm{i}}=F_{\mathrm{i}} \Omega_{\mathrm{b}}$ for various classes of objects. We display in Table 1 a comparison of our results with observational estimates by Fukugita et al. (1998). We note that our predictions are consistent with the data. In Table 1 we removed from the mass embedded within clusters and groups shown in Fig. 13 the fraction of baryons in stars or galactic cores which they contain.

\section{Open universe}

Using the same formalism we can also study the case of an open universe $\Omega_{\mathrm{m}}=0.3, \Omega_{\Lambda}=0$, with a CDM power spectrum normalized to $\sigma_{8}=0.77$. We use $\Omega_{\mathrm{b}}=0.03$ and $H_{0}=60 \mathrm{~km} \mathrm{~s}^{-1} / \mathrm{Mpc}$.

\subsection{Galaxies}

We show in Fig. 14 the correlation function of galaxies brighter than $M_{B}=-18.6$ at $z=0$ (upper solid line). Our predictions are similar to the results obtained in Fig. 3. However, a bump appears at intermediate scales $(R \sim 2 \mathrm{Mpc})$ where $\bar{\xi} \sim 10$. This corresponds to the sharp increase of $\bar{\xi}(R)$ from the linear regime $(\bar{\xi} \lesssim 1)$ to the nonlinear regime $(\bar{\xi} \gtrsim 100)$. This leads to a "step-like" profile which is also seen in numerical simulations (e.g., Valageas et al. 2000) and which gets larger for low density universes (Peacock \& Dodds 1996). This is due to the slow-down of the linear growth factor while the two-point correlation function keeps increasing as $\bar{\xi}(R, a) \propto a^{3}$ in the highly non-linear regime (Valageas \& Schaeffer 1997). The observed two-point correlation function on the other hand is surprisingly close to a power-law as soon as $\bar{\xi} \geq 1$. Such a behaviour requires a precise balance between the curvature of the initial power-spectrum, the distortions induced by the non-linear dynamics and the bias of galaxies (which also depends on non-gravitational processes which affect galaxy formation). Note however that using numerical simulations Pearce et al. (1999) and Benson et al. (2000a) obtain a good match to observations as the galaxies they get are antibiased relative to the dark matter on small scales which "conspires" to provide a power-law galaxy correlation function. This behaviour is partly due to the spatial exclusion of halos. Moreover, the expression (5) we use for the bias is only valid at large separations $r \gg R$ between objects of size $R$. Thus, the discrepancy at small scales might be cured by a more rigorous calculation which would not use the approximation $r \gg R$.

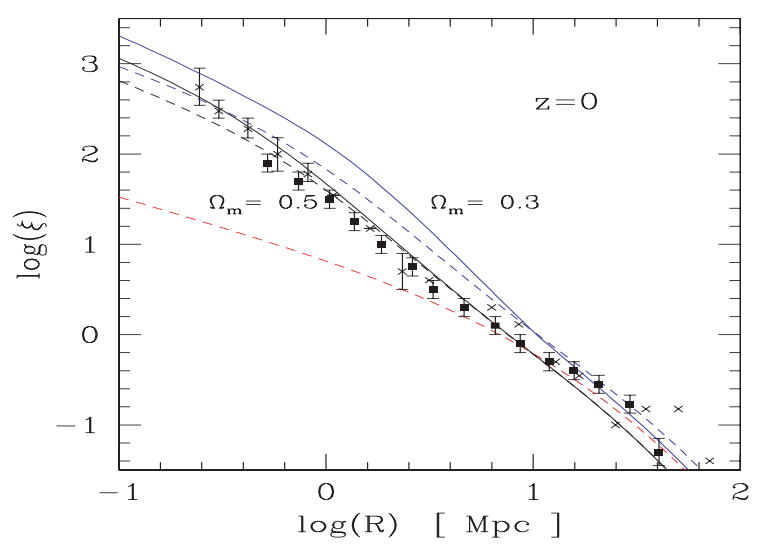

Fig. 14. The correlation function of galaxies brighter than $M_{B}=-18.6$ at $z=0$ as in Fig. 3. The upper solid line corresponds to the open universe with $\Omega_{\mathrm{m}}=0.3$ and $\Omega_{\Lambda}=0$, while the lower dashed-line is the linear prediction. The upper dashed line shows the low-density flat universe with $\Omega_{\mathrm{m}}=0.3$ and $\Omega_{\Lambda}=0.7$. The middle solid line (resp. dashed line) displays the case $\Omega_{\mathrm{m}}=0.5$ and $\Omega_{\Lambda}=0$ (resp. $\Omega_{\mathrm{m}}=0.5$ and $\left.\Omega_{\Lambda}=0.5\right)$

On the other hand, note that in the case of a critical density universe this "bump" in the mildly non-linear regime is much smaller, which leads to good agreement with observations (see Fig. 3). In particular, we also display in Fig. 14 the dark matter correlation function we would obtain for a low-density flat universe (upper dashed line) with $\Omega_{\mathrm{m}}=0.3$ and $\Omega_{\Lambda}=0.7$, with a slightly different power-spectrum from Bardeen et al. (1989) (and $\Gamma=0.18)$ using the non-linear fit from Peacock \& Dodds 1996). Thus this corresponds to a bias of unity, which is a good approximation for galaxies with $M_{B} \leq-18.6$ at $z=0$, see Figs. 1 and 15 . The main point is that the "bump" is much weaker for a flat cosmology, as can also be seen in Peacock \& Dodds (1996). The intermediate lines show the dark matter correlation functions obtained for $\Omega_{\mathrm{m}}=0.5$ (with $\Omega_{\Lambda}=0$ and $\Omega_{\Lambda}=0.5$ ) and normalized to $\sigma_{8}=0.6$ (and $\Gamma=0.3$ ). Thus, the shape itself of the galaxy correlation function does not allow one to strongly discriminate between these various cosmological scenarios. Although the "bump" which appears for the open universe with $\Omega_{\mathrm{m}}=0.3$ seems to disfavour this model, the inaccuracy involved in the modelling of the galaxy clustering properties is certainly too large to draw meaningful conclusions, especially since numerical simulations suggest that simple physical processes could remove this feature (Benson et al. 2000a). 


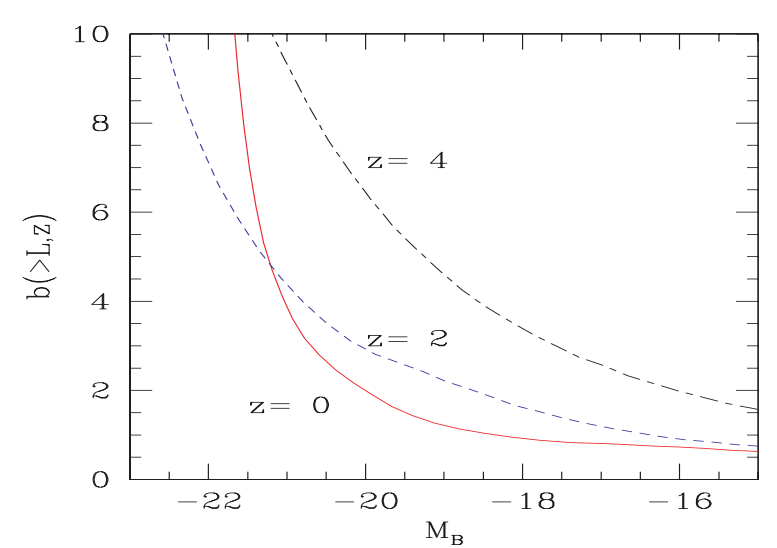

Fig. 15. The redshift evolution of the relation luminosity-bias. The solid line corresponds to $z=0$, the dashed line to $z=2$ and the dot-dashed line to $z=4$

We present in Fig. 15 the redshift evolution of the luminosity-bias relation. We can see that our results are again similar to those obtained in Fig. 4, since the astrophysical model is the same and it must satisfy the same observational constraints at low $z$. However, the bias of bright galaxies increases more slowly with redshift than for a critical density universe (compare the curve at $z=4$ with Fig. 4). This is due to the higher normalization $\sigma_{8}$ of the power-spectrum and to the slower redshift evolution of the linear growth factor which means that massive galactic halos correspond to less extreme density fluctuations at $z=4$ than for the case $\Omega_{\mathrm{m}}=1$ when they have a similar clustering pattern at $z=0$.

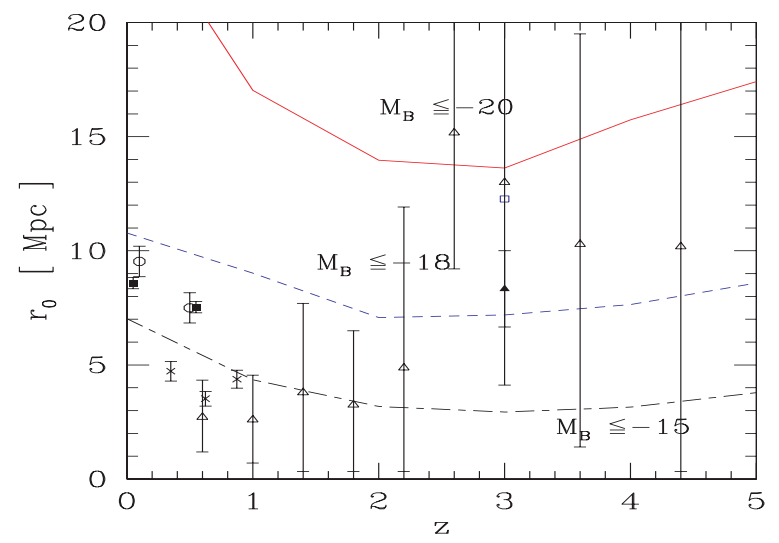

Fig. 16. The redshift evolution of the correlation length $r_{0}$ (in comoving Mpc) associated with galaxies below various $B$-band magnitude thresholds, as in Fig. 6, for an open universe

We show in Fig. 16 the redshift evolution of the comoving correlation length $r_{0}$ associated with galaxies below various $B$-band magnitude thresholds. The galaxy correlation length is larger than for the previous case of a critical density universe because the normalization of the powerspectrum $\left(\sigma_{8}\right)$ is slightly higher. This also implies a slower redshift evolution. We note that the clustering pattern we predict for Lyman break galaxies is somewhat too strong as compared to observations. However, this could be due
Table 2. Baryonic parameter $\Omega_{\mathrm{i}}$ associated with various objects at $z=0$ and $z=3$, for an open universe with $\Omega_{\mathrm{m}}=0.3$, $\Omega_{\mathrm{b}}=0.03$. The second line which appears for some contributions is the observational estimate from Fukugita et al. (1998)

\begin{tabular}{ccc}
\hline & $z=0$ & $z=3$ \\
\hline \hline stars & 0.0039 & 0.0006 \\
& $0.002-0.0057$ & \\
\hline galactic gas & 0.019 & 0.013 \\
\hline groups & 0.011 & 0.0007 \\
& $0.011-0.045$ & \\
\hline clusters & 0.003 & 0.00002 \\
& $0.0012-0.0044$ & \\
\hline Lyman- $\alpha$ forest & 0.007 & 0.016 \\
& & $0.01-0.05$ \\
\hline
\end{tabular}

to some extinction by dust of the luminosity of these LBG. Indeed, in our model the number of galaxies brighter than $M_{B}=-18$ is $n=310^{-3} \mathrm{Mpc}^{-3}$ while the abundance of LBG is $n \sim 8.510^{-4} \mathrm{Mpc}^{-3}$ (Adelberger et al. 1998). This means that we would recover the right correlation length if we assumed that LBG correspond to $30 \%$ of these galaxies with $M_{B} \leq-18$. An alternative would be that LBG correspond to galaxies which are in the midst of a starburst phase (e.g., Somerville et al. 2000) which increases their luminosity (note that the absolute LBG magnitude is rather large: $M_{R} \leq-21.8$ for an apparent magnitude $\mathcal{R} \leq 25.5$ without K-correction). In this case, Lyman-break galaxies would also be drawn from a larger parent population which could again lead to a smaller correlation length. Note on the other hand that it would be difficult to reconcile the model with observations if we had predicted a smaller value of $r_{0}$ than the one given by the data.

\subsection{Distribution of matter}

We show in Fig. 17 the contributions of stars, galactic gas and Lyman- $\alpha$ clouds to the baryonic density in the universe as a function of redshift. We obtain a behaviour similar to Fig. 12.

We display in Fig. 18 the redshift evolution of the fraction of matter embedded within galaxies, groups, clusters and stars. We see that the mass fraction formed by groups and clusters declines more slowly than in Fig. 13. Again this is due to the higher normalization $\sigma_{8}$ and to the slower redshift evolution of the linear growth factor.

We show in Table 2 the baryonic density parameters $\Omega_{\mathrm{i}}$ associated with various objects. We note that our results still agree reasonably well with observations.

\section{Conclusion}

In this article we have described the redshift evolution of the clustering of various objects (galaxies, quasars, Lyman- $\alpha$ clouds, clusters) within the framework of a unified model of structure formation which has already been checked against many observations. Hence there are no 


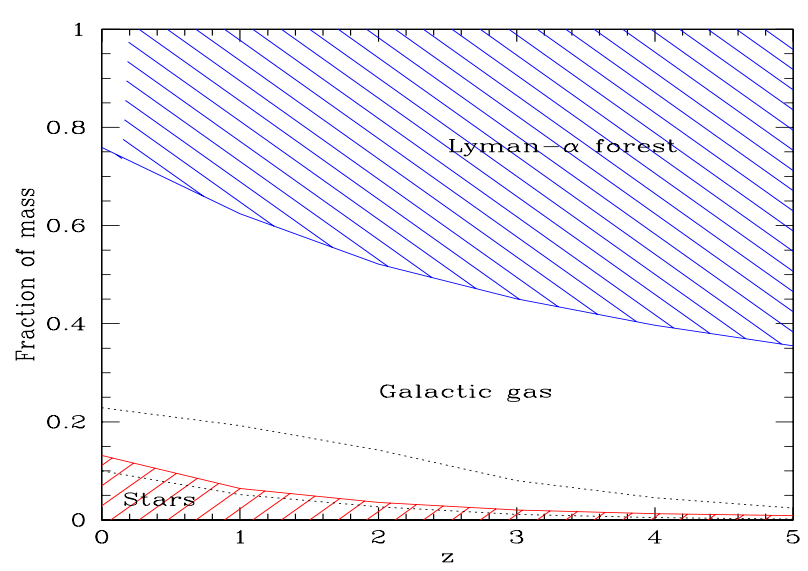

Fig. 17. The redshift evolution of the fraction of baryonic mass embedded within Lyman- $\alpha$ forest clouds (upper shaded area), galactic halos (white area) and stars (lower shaded area). The dotted lines separate the contributions from bright $\left(M_{B}<-19.5\right.$, lower part) and faint $\left(M_{B}>-19.5\right.$, upper part $)$ galaxies for stars and gas

free parameters chosen to fit the observations considered in this article, since the model was already overconstrained by previous studies. We have shown that the bias of these astrophysical objects displays an intricate behaviour which cannot be described by simple power-law parameterizations. Indeed, the link between these objects and the underlying dark matter component reflects the influence of many factors (cooling processes, star formation efficiency, evolution of the UV background radiation field...) which can have competing effects. We have shown that in our model the bias associated with galaxies strongly increases with their luminosity. This was already emphasized in the earlier calculations (Schaeffer 1987; Bernardeau \& Schaeffer 1992) as being a genuine outcome of the hierarchical clustering hypothesis. We recover the trend seen in observations, although our dependence may be slightly too strong. However, this could be due to the simplicity of our model which does not include any scatter in the properties of the objects we model. The redshift evolution we obtain also agrees with data, in particular the correlation length of Lyman-break galaxies observed at $z=3$ is consistent with our results.

We have also described the bias associated with Lyman- $\alpha$ clouds. Thus, it appears that absorbers in the range $10^{16}<N_{\mathrm{HI}}<10^{21} \mathrm{~cm}^{-2}$ have a bias close to unity (hence they trace the dark matter density field) in contrast to other clouds and galaxies which show a very different behaviour of their clustering properties. Our results also agree with observations for quasars and clusters. In particular, they explain in a natural fashion the qualitative and quantitative differences between the redshift evolution of the clustering of QSOs and of galaxies. Thus, our model provides a good description of the intrinsic properties and the spatial clustering of a wide variety of objects, from small underdense Lyman- $\alpha$ absorbers up to massive clusters, which covers six orders of magnitude in mass.

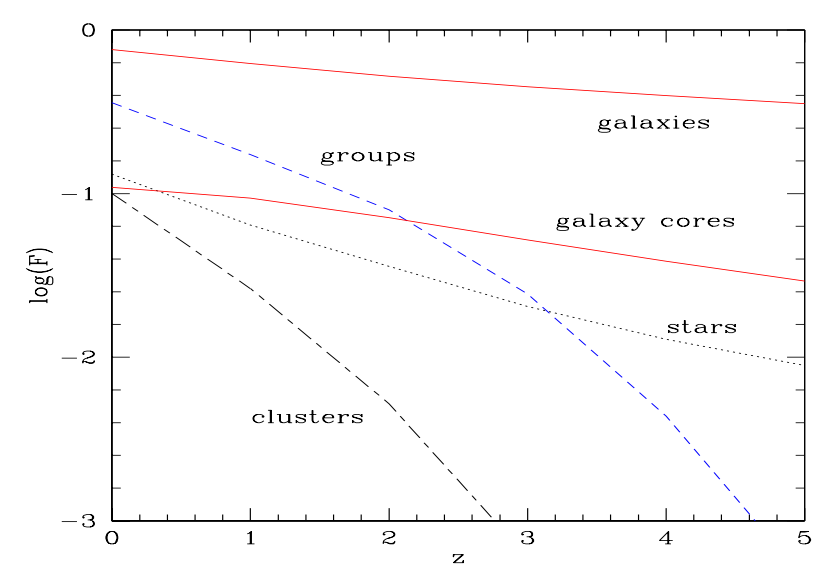

Fig. 18. The redshift evolution of the fraction $F$ of baryonic mass embedded within galaxies (upper solid line: total mass, lower solid line: cores), groups (dashed line), clusters (dotdashed line) and stars (dotted line)

Finally, we have described the redshift evolution of the distribution of matter over the various objects observed in the universe. Our results show a reasonable agreement with observations. This is not surprising since our model has already been checked against the galaxy luminosity function, the number density of Lyman- $\alpha$ clouds, which constrain the amount of matter embedded within these objects. In particular, it appears that light does not trace baryonic mass as bright galaxies $\left(M_{B}<-19.5\right)$ which contain most of the stars only provide a small fraction of the mass associated with virialized and cooled halos.

We have also performed the same analysis for the case of an open universe. In both cosmologies our results agree reasonably well with observations but the details of astrophysical models are still too uncertain to discriminate between these two cases. However, for the open universe the galaxy correlation function we obtain shows a shoulder which does not appear in observations. This could be due to the approximation involved in our calculation which is only valid on scales much larger than the size of the objects. In particular, some numerical simulations do not get this "bump" (Benson et al. 2000a). On the other hand, they do not recover the luminosity dependence of the galaxy bias (Benson et al. 2000b) and simulations cannot simultaneously describe the clustering of the whole range of objects we studied in this paper. Nevertheless, the fact that our predictions are nearly consistent with the data over such a wide range of objects shows that hierarchical scenarios provide a very good description of structure formation. On the other hand, more detailed observations of the amplitude of the clustering of galaxies selected by their luminosity will constrain the astrophysical models.

Acknowledgements. Much of this work was performed at the Center for Particle Astrophysics, University of California, Berkeley, whose hospitality we gratefully acknowledge. 


\section{References}

Adelberger, K. L., Steidel, C. C., Giavalisc, M., et al. 1998, ApJ, 505, 18

Balian, R., \& Schaeffer, R. 1989, A\&A, 220, 1

Bardeen, J. M., Bond, J. R., Kaiser, N., \& Szalay, A. S. 1986, ApJ, 304, 15

Baugh, C. M. 1996, MNRAS, 280, 267

Baugh, C. M., Benson, A. J., Cole, S., Frenk, C. S., \& Lacey, C. G. 1999, MNRAS, 305, L21

Benoist, C., Maurogordato, S., da Costa, L. N., Cappi, A., \& Schaeffer, R. 1996, ApJ, 472, 452

Benson, A. J., Cole, S., Frenk, C. S., Baugh, C. M., \& Lacey, C. G. 2000a, MNRAS, 311, 793

Benson, A. J., Cole, S., Frenk, C. S., Baugh, C. M., \& Lacey, C. G. 2000b, MNRAS, 316, 107

Bernardeau, F., \& Schaeffer, R. 1991, A\&A, 220, 23

Bernardeau, F., \& Schaeffer, R. 1992, A\&A, 255, 1

Bernardeau, F., \& Schaeffer, R. 1999, A\&A, 349, 697

Bouchet, F. R., Schaeffer, R., \& Davis, M. 1991, ApJ, 383, 19

Borgani, S., Plionis, M., \& Kolokotronis, V. 1999, MNRAS, 305,866

Carlberg, R. G., Yee, H. K. C., Morris, S. L., et al. 1998, Royal Society Discussion Meeting [astro-ph 9805131]

Colombi, S., Bouchet, F. R., \& Schaeffer, R. 1992, A\&A, 263, 1

Colombi, S., Bouchet, F. R., \& Schaeffer, R. 1994, A\&A, 281, 301

Colombi, S., Bouchet, F. R., \& Schaeffer, R. 1995, ApJS, 96, 401

Colombi, S., Bernardeau, F., Bouchet, F. R., \& Hernquist, L. 1997, MNRAS, 287, 241

Croft, R. A. C., Dalton, G. B., Efstathiou, G., Sutherland, W. J., \& Maddox, S. J. 1997, MNRAS, 291, 305

Dave, R., Hernquist, L., Katz, N., \& Weinberg, D. H. 1999, ASP Conf. Ser. [astro-ph 9910221]

Davis, M., Efstathiou, G. P., Frenk, C. S., \& White, S. D. M. 1985, ApJ, 292, 371

Fukugita, M., Hogan, C. J., \& Peebles, P. J. E. 1998, ApJ, 503, 518

Kauffmann, G., Colberg, J. M., Diaferio, A., \& White, S. D. M. 1999, MNRAS, 303, 188
La Franca, F., Andreani, P., \& Cristiani, S. 1998, ApJ, 497, 529

Le Fevre, O., Hudon, D., Lilly, S. J., et al. 1996, ApJ, 461, 534

Loveday, J., Maddox, S. J., Efstathiou, G., \& Peterson, B. A. 1995, ApJ, 442, 457

Magliocchetti, M., \& Maddox, S. J. 1999, MNRAS, 306, 988

Mo, H. J., \& White, S. D. M. 1996, MNRAS, 282, 347

Munshi, D., Bernardeau, F., Melott, A. L., \& Schaeffer, R. 1999a, MNRAS, 303, 433

Munshi, D., Coles, P., \& Melott, A. L. 1999b, MNRAS, 307, 387

Munshi, D., Coles, P., \& Melott, A. L. 1999c, MNRAS, 310, 892

Peacock, J. A., \& Dodds, S. J. 1996, MNRAS, 280, L19

Peacock, J. A., \& Smith, R. E. 2000, submitted to MNRAS [astro-ph 0005010]

Pearce, F. R., Jenkins, A., Frenk, C. S., et al. 1999, ApJ, 521, L99

Peebles, P. J. E. 1982, ApJL, 263, L1

Ponman, T. J., Cannon, D. B., \& Navarro, J. F. 1999, Nature, 397,135

Postman, M., Lauer, T. R., Szapudi, I., \& Oegerle, W. 1998, ApJ, 506, 33

Press, W. H., \& Schechter, P. 1974, ApJ, 187, 425

Schaeffer, R. 1984, A\&A, 134, L5

Schaeffer, R. 1985, A\&A, 144, L1

Schaeffer, R. 1987, A\&A, 180, L5

Seljak, U. 2000, Phys. Rev. D, submitted [astro-ph 0001493]

Somerville, R. S., Primack, J. R., \& Faber, S. M. 2000, MNRAS, accepted [astro-ph 0006364]

Stephens, A. W., Schneider, D. P., Schmidt, M., et al. 1997, AJ, 114, 41

Valageas, P., \& Schaeffer, R. 1997, A\&A, 328, 435

Valageas, P. 1999, A\&A, 347, 757

Valageas, P., \& Schaeffer, R. 1999, A\&A, 345, 329

Valageas, P., \& Silk, J. 1999a, A\&A, 347, 1

Valageas, P., \& Silk, J. 1999b, A\&A, 350, 725

Valageas, P., Schaeffer, R., \& Silk, J. 1999, A\&A, 345, 691

Valageas, P., \& Schaeffer, R. 2000, A\&A, 359, 821

Valageas, P., Lacey, C., \& Schaeffer, R. 2000, MNRAS, 311, 234

Willmer, C. N. A., da Costa, L. N., \& Pellegrini, P. S. 1998, AJ, 115,869 